\title{
A mathematical model for virus infection in a system of interacting computers
}

\author{
J. LÓPEZ GONDAR and R. CIPOLATTI \\ Departamento de Matemática Aplicada \\ Instituto de Matemática, Universidade Federal do Rio de Janeiro \\ C.P. 68530 , Rio de Janeiro, Brasil \\ E-mail: juan@labma.ufrj.br / cipolatti@im.ufrj.br
}

\begin{abstract}
We introduce a simplified theoretical model to describe a virtual virus propagation process in a set of interacting computers. The propagation mechanisms considered here are those related to the reception of messages through internet as well as the ones concerning the simple exchange of files using recording devices as compact disks or the commonly used floppy disks. In spite of its inherent simplicity, this model provides a good idea of the infection process and trends. From the mathematical point of view, the nonlinear delay integral equation that we obtain here presents certain interesting features which are explored and enlightened in this paper.
\end{abstract}

Mathematical subject classification: 45G10, 49K25, 92D99.

Key words: virtual viruses, nonlinear delay integral equation.

\section{Introduction}

The infection of computers by virtual viruses is a present day problem. A lot of efforts have been (and will be) devoted to the development of virtual vaccines each time a new virus appears. Nevertheless, slow progress (if any) in understanding the propagation process, quantitatively, has been obtained up to now. In a certain sense, the propagation of virtual viruses in a system of interacting computers could be compared with a disease transmitted by vectors when dealing with public health. Concerning diseases transmitted by vectors, one has to take into account that the parasite spends part of its lifetime inhabiting the vector, so that the infection switches back and forth between host and vector. Almost the same 
occurs here and also a certain time interval elapses between successive contacts which, in this case, involves an electronic message (or a recording device) and two different computers. However, we have to point out that, in our simplified model, which ignores finer details, we suppose in the case of recording devices that they are simple carriers not permanently infected, which could transmit the virus only once (if it is so); i.e., after the first transmission of a certain program, the contents in the recording device are removed before it is used again. Under such hypothesis (among others considered below) we obtain a compact model restricted to one single integral equation.

The paper is organized as follows: in Section 2, we construct the theoretical model. Section 3 is devoted to performing a mathematical analysis of the integral equation obtained: existence, uniqueness and asymptotic behavior of the solutions, as well as stability of stationary solutions. Finally, Section 4 contains numerical experiments and our conclusions.

\section{Modeling the dynamics of transmission}

Before introducing the new model to describe the way by which some virtual virus infection transmitted by electronic messages (or by recording devices) could spread through a population of computers as a function of time, we shall start deducing a certain contact-propagation equation for a general infectious process having a natural (or induced) recovering time. We consider such an equation as a convenient point of reference for subsequent analysis. In the present approach we shall assume to deal with a new kind of virus infection for which there exists no vaccine available. Being so, the unique way to restore the functions of an infected computer is by deleting the hard disk (with which it becomes susceptible again). It should be pointed out that neither spatial dependence nor latent periods or immunity factors will be considered here.

Therefore, the total population is divided into two classes:

(I) the infective class. This includes all infected individuals because, in the absence of a latent period, any infected individual immediately becomes an infective one, displaying a potential capacity of transmitting the disease to other susceptible individuals; 
(S) the susceptible class; i.e., those individuals capable of contracting the disease, becoming themselves infected.

The closed host population of total size $N$ will be used as a normalization factor, so we set $I+S=1$.

Let us denote by $g(\tau)$ the rate of the infection process at time $\tau$, i.e., the number of new infected computers (population density of them) per unit time, at instant $\tau$. We also introduce a function of two variables $P(t, \tau), P: \Lambda \rightarrow[0,1]$, where

$$
\Lambda=\left\{(t, \tau) \in \mathbb{R}^{2} ; t \geq \tau\right\}
$$

which represents the probability of a computer infected at $\tau$ to remain infectious at $t$. Such a function, of course, is related to the time elapsed between the detection of the infection and its eradication by erasing the hard disk. Considering a maximum recovering time $T$, the function $P(t, \tau)$ is supposed to have the following general properties:

i) $P(t, \tau)=1 \quad$ if $\tau=t$;

ii) $P(t, \tau)=0 \quad$ if $\quad \tau \leq t-T$;

iii) $P(t, \tau)$ is a monotone decreasing function of $t$ for each fixed $\tau$.

If we want to describe the density of infective individuals as a function of time, starting at $t=0$, the only infected ones to be considered should be those in the interval $[-T, t]$ (just because of the maximum recovering time $T$ ).

Dividing this interval into $n$ equal parts, so that $\Delta \tau=(t+T) / n$ and defining $\tau_{1}=-T, \tau_{2}=-T+\Delta \tau, \ldots, \tau_{n+1}=-T+n \Delta \tau$, i.e., $\tau_{j}=-T+(j-1) \Delta \tau$, $j=1, \ldots, n+1$, we should state, for $\Delta \tau$ small enough, that the number of infected individuals in the interval $\left[\tau_{j}, \tau_{j+1}\right]=\left[\tau_{j}, \tau_{j}+\Delta \tau\right]$ is given, approximately, as

$$
g\left(\tau_{j}\right)\left(\tau_{j+1}-\tau_{j}\right)=g\left(\tau_{j}\right) \Delta \tau, \quad j=1,2, \ldots, n .
$$

Then, the number of infected individuals in this interval which remain infective at $t$ is approximately,

$$
g\left(\tau_{j}\right) P\left(t, \tau_{j}\right) \Delta \tau, \quad j=1,2, \ldots, n,
$$


and therefore, the total number of infective individuals at $t$ may be written, approximately, as

$$
\sum_{j=1}^{n} g\left(\tau_{j}\right) P\left(t, \tau_{j}\right) \Delta \tau,
$$

which, in the limit $\Delta \tau \rightarrow 0$, gives

$$
I(t)=\int_{-T}^{t} g(\tau) P(t, \tau) d \tau, \quad t \geq 0 .
$$

Taking into account that $P(t, \tau)=0$ if $\tau \leq t-T$, we should write Eq. (2.6) as

$$
I(t)=\int_{t-T}^{t} g(\tau) P(t, \tau) d \tau, \quad t \geq 0 .
$$

The former equation contains the basic features for the evolution of an infection process in the framework of a SIS model. Depending on the specific transmission process, one should conceive, in each case, a suitable infection rate-function $g(\tau)$. As it can be verified in references [4, 6], Eq. 2.7 and variations of it have already been used by other authors in describing the propagation of diseases.

Concerning the propagation of virtual viruses transmitted by electronic messages (a) or by recording devices (b), we shall establish the following hypothesis to construct $g(\tau)$ :

a) some finite time elapses between the act of sending and the reception of messages ( $t_{0}$ on average);

b1) each recording device is used only twice in intervals of time $t_{0}$, on average;

b2) each device transmits the infection when, used by the first time in an infected computer, it is used by the second time in a susceptible one. Once this process occurs, previous contents are deleted.

Possible events concerning consecutive records and its corresponding probabilities are described below. 


\begin{tabular}{|c|c|}
\hline Event & Probability \\
\hline$I \mapsto I$ & $I(t) I\left(t+t_{0}\right)$ \\
\hline$S \mapsto S$ & $S(t) S\left(t+t_{0}\right)=(1-I(t))\left(1-I\left(t+t_{0}\right)\right)$ \\
\hline$I \mapsto S$ & $I(t) S\left(t+t_{0}\right)=I(t)\left(1-I\left(t+t_{0}\right)\right)$ \\
\hline$S \mapsto I$ & $S(t) I\left(t_{0}\right)=(1-I(t)) I\left(t+t_{0}\right)$ \\
\hline
\end{tabular}

Among them we are only interested in the third one.

By a direct calculation, one can easily verify that the sum of the above probabilities gives just 1 .

Of course, there exists some characteristic average frequency for the submission of electronic messages in the system of interacting computers considered here, as well as recording devices are subjected to a certain recording frequency. We shall denote such a characteristic frequency by $N_{r}(t)$. Then, for a time interval $\Delta t$ small enough, we may write the number of new infected computers which are increased to those already existing at $t+t_{0}$, approximately, by

$$
\left.\Delta N_{I}\right|_{t+t_{0}}=N_{r}(t) \Delta t I(t)\left(1-I\left(t+t_{0}\right)\right)
$$

Dividing by $N \Delta t$ and taking the limit as $\Delta t \rightarrow 0$, we have

$$
g\left(t+t_{0}\right)=\frac{N_{r}(t)}{N} I(t)\left(1-I\left(t+t_{0}\right)\right) .
$$

If we make the substitution $t \mapsto t+t_{0}$, Eq. (2.9) may be written as

$$
g(t)=\frac{N_{r}\left(t-t_{0}\right)}{N} I\left(t-t_{0}\right)(1-I(t)),
$$

or

$$
g(t)=\alpha(t) I\left(t-t_{0}\right)(1-I(t))
$$

where

$$
\alpha(t)=\frac{N_{r}\left(t-t_{0}\right)}{N} .
$$


Therefore, in the framework of our model and following Eq. (2.7), the density of infective computers at time $t$ due to the virtual virus propagation process obeys the following law

$$
I(t)=\int_{t-T}^{t} \alpha(\tau) I\left(\tau-t_{0}\right)(1-I(\tau)) P(t, \tau) d \tau, \quad t>0,
$$

where $\alpha(\tau)$ is given by (2.12).

Eq. (2.13) is a delay integral equation and the existence of solutions for $t \geq 0$ depends on data defined in the past $-T-t_{0} \leq t \leq 0$.

Although we are only considering $t_{0}>0$, it is interesting to point out that, if $t_{0}=0$ and $P(t, \tau)=\phi(t-\tau)$, where $\phi(\xi)$ is a real function, Eq. (2.13) reduces to the special case considered by Cooke and Yorke in [6].

\section{Existence, uniqueness and asymptotic behavior}

In this section we consider the problem of existence, uniqueness and asymptotic behavior of solutions for equation (2.13). We will say that a function $I(t)$ is a solution generated by $I_{0}$ if $I(t)=I_{0}(t)$ a.e. in $\left[-T-t_{0}, 0\right]$ and if $I(t)$ also satisfies (2.13) for $t>0$.

\section{- Uniqueness:}

We consider the set $\Lambda$ defined by (2.1), a function $P: \Lambda \rightarrow[0,1]$ such that (2.2) holds and $I_{0} \in L^{\infty}\left(-T-t_{0},+\infty\right)$.

In order to avoid technicalities (see Remark 3.2), we assume that

$$
\alpha \in C\left(\left[-T-t_{0},+\infty[) .\right.\right.
$$

Lemma 3.1. Assume that $\alpha$ satisfies (3.1). If $I_{1}$ and $I_{2}$ are solutions of (2.13) generated by $I_{0}(t)$, then $I_{1}(t)=I_{2}(t)$ for all $t>0$.

Proof. First of all it should be noticed that, if $I_{i}$ is a solution of (2.13), then $I_{i}$ is continuous in $] 0,+\infty[$.

For $0<t \leq t_{0}$ we have

$$
I_{1}(t)-I_{2}(t)=\int_{t-T}^{t} \alpha(\tau) I_{0}\left(\tau-t_{0}\right)\left(I_{2}(\tau)-I_{1}(\tau)\right) P(t, \tau) d \tau .
$$


Let $\varphi$ be the function defined by $\varphi(t)=\left|I_{1}(t)-I_{2}(t)\right|$. Since $0 \leq P(t, \tau) \leq 1$, it follows that

$$
\varphi(t) \leq\left\|I_{0}\right\| \int_{0}^{t}|\alpha(\tau)| \varphi(\tau) d \tau, \quad 0<t \leq t_{0},
$$

where $\left\|I_{0}\right\|=\operatorname{ess} \sup \left\{\left|I_{0}(\tau)\right| ;-T-t_{0} \leq \tau \leq 0\right\}$.

Defining

$$
\psi(t)=\int_{0}^{t}|\alpha(\tau)| \varphi(\tau) d \tau,
$$

it follows that $\psi$ is differentiable in $] 0,+\infty\left[\right.$ and we have $\psi^{\prime}(t)=|\alpha(t)| \varphi(t)$.

After multiplying both sides of (3.2) by $|\alpha(t)|$, we get

$$
\psi^{\prime}(t)-\left\|I_{0}\right\||\alpha(t)| \psi(t) \leq 0, \quad 0<t<t_{0},
$$

from which we obtain

$$
\frac{d}{d t}\left[\psi(t) \exp \left(-\left\|I_{0}\right\| \int_{0}^{t}|\alpha(\tau)| d \tau\right)\right] \leq 0, \quad 0<t<t_{0} .
$$

Since $\psi(0)=0$, we conclude that $\psi(t)=0$ as well as $\varphi(t)=0$ for all $0<t \leq t_{0}$.

The same arguments can be repeated for $(k-1) t_{0}<t \leq k t_{0}$, for all $k \in \mathbb{N}$, and the proof is achieved.

Remark 3.2. We can obtain the same result of Lemma 3.1 with essentially the same proof under the weaker condition

$$
\alpha \in L_{\mathrm{loc}}^{1}\left(-T-t_{0},+\infty\right) .
$$

\section{- Existence and asymptotic behavior:}

In order to prove the existence of solutions for Eq. (2.13), we assume that

$$
\alpha \in L_{\mathrm{loc}}^{1}\left(-T-t_{0},+\infty\right), \quad \alpha(t) \geq 0 .
$$

In view of the model we have in mind, we restrict our analysis to the solutions that satisfy $0 \leq I(t) \leq 1$, which allows us to consider the space $E=L^{\infty}(]-T-t_{0},+\infty[)$. This is a Banach space for the norm

$$
\|f\|=\operatorname{ess} \sup \left\{|f(\tau)| ; \tau \geq-T-t_{0}\right\} .
$$


In the sequel, we distinguish two cases, referred here as "Slow Infective Rate Case" and "General Infective Rate Case', respectively.

Let $r(t)$ be the function defined by

$$
r(t)=\int_{t-T}^{t} \alpha(\tau) P(t, \tau) d \tau .
$$

Case 1: "Slow Infective Rate" : $r(t) \leq 1, \quad \forall t \geq 0$.

For a given $I_{0} \in E$ such that $0 \leq I_{0}(t) \leq 1$, we consider the set

$$
E\left(I_{0}\right)=\left\{I \in E ; 0 \leq I(t) \leq 1, I(t)=I_{0}(t), \text { a.e. in }\left[-T-t_{0}, 0\right]\right\},
$$

which is a closed subset of $E$. We also consider the operator $\Phi: E\left(I_{0}\right) \rightarrow E\left(I_{0}\right)$ defined as follows: $\Phi[I](t)=I_{0}(t)$ almost everywhere in $\left[-T-t_{0}, 0\right]$ and, for $t>0$,

$$
\Phi[I](t)=\int_{t-T}^{t} \alpha(\tau) I\left(\tau-t_{0}\right)(1-I(\tau)) P(t, \tau) d \tau .
$$

The next theorem establishes the existence of a solution of Eq. (2.13) in $E$ (which is unique from Lemma 3.1), as well as its asymptotic behavior in the case $r(t)<1$ for $t$ large enough.

Theorem 3.3. Assume $\alpha$ satisfies (3.3) and $r(t) \leq 1, \forall t \geq 0$. If $I_{0} \in E$ is such that $0 \leq I_{0}(t) \leq 1$, then the operator $\Phi$ defined by (3.6) has a unique fixed point $\bar{I} \in E\left(I_{0}\right)$. In addition, if

$$
\limsup _{t \rightarrow+\infty} r(t)<1
$$

then there exist $C, M, L>0$ such that

$$
\bar{I}(t) \leq C e^{-L t}, \quad \forall t \geq M .
$$

Proof. We divide the proof into two steps. Let $a, b$ be two real numbers such that $-T-t_{0}<a<b$ and consider the set

$$
\begin{array}{r}
E_{a, b}\left(I_{0}\right)=\left\{I \in E ; 0 \leq I(t) \leq 1, I(\tau)=I_{0}(\tau),\right. \\
\text { for } \tau<a, \text { and } I(\tau)=0, \text { for } \tau>b\} .
\end{array}
$$


It is evident that $E_{a, b}\left(I_{0}\right)$ is a nonempty closed subset of $E$ and, for $a \geq 0$, $E_{a, b}\left(I_{0}\right) \subset E\left(I_{0}\right)$.

We introduce the operator $\Phi: E_{a, b}\left(I_{0}\right) \rightarrow E_{a, b}\left(I_{0}\right)$ defined by $\Phi[I](t)=I_{0}(t)$ for $t \leq a, \Phi[I]=0$ for $t>b$ and

$$
\left.\left.\Phi[I](t)=\int_{t-T}^{t} \alpha(\tau) I\left(\tau-t_{0}\right)(1-I(\tau)) P(t, \tau) d \tau . \quad \forall t \in\right] a, b\right] .
$$

Step 1: Let $I, \widetilde{I} \in E_{0, b}\left(I_{0}\right)$ and consider the operator $\Phi$ defined in (3.8), with $a=0$ and

$$
b=\min \left\{t_{0}, T\right\} .
$$

Then, it is easily seen that $\Phi[I](t)-\Phi[\tilde{I}](t)=0$ a.e. in $\left[-T-t_{0}, 0\right]$ and

$$
\begin{gathered}
\Phi[I](t)-\Phi[\tilde{I}](t)=\int_{0}^{t} \alpha(\tau) I_{0}\left(\tau-t_{0}\right)(\tilde{I}(\tau)-I(\tau)) P(t, \tau) d \tau \\
\forall t \in] 0, b] .
\end{gathered}
$$

Since $0 \leq P(t, \tau) \leq 1$ for all $(t, \tau) \in \Lambda$, it follows that

$$
|\Phi[I](t)-\Phi[\tilde{I}](t)| \leq\left\|I_{0}\right\|\|\tilde{I}-I\| \int_{0}^{t} \alpha(\tau) d \tau
$$

for every $t \in] 0, b]$. A recurrent argument using (3.10) and (3.11) gives, for $t \in] 0, b]$,

$$
\left|\Phi^{k}[I](t)-\Phi^{k}[\tilde{I}](t)\right| \leq \frac{1}{k !}\left\|I_{0}\right\|^{k}\left(\int_{0}^{t} \alpha(\tau) d \tau\right)^{k}\|\tilde{I}-I\|,
$$

from which we obtain easily

$$
\left\|\Phi^{k}[I]-\Phi^{k}[\tilde{I}]\right\| \leq \frac{1}{k !}\left\|I_{0}\right\|^{k}\left(\int_{0}^{b} \alpha(\tau) d \tau\right)^{k}\|\tilde{I}-I\| .
$$

Choosing $k$ large enough, it follows from (3.13) that $\Phi^{k}$ is a contraction in $E_{0, b}\left(I_{0}\right)$ and the Banach fixed point theorem assures the existence of a unique fixed point $I_{1}$ for $\Phi^{k}$ in $E_{0, b}\left(I_{0}\right)$. More precisely, there exists a unique $I_{1} \in$ $E_{0, b}\left(I_{0}\right)$ such that $\Phi^{k}\left[I_{1}\right]=I_{1}$. Since $\Phi^{k+1}\left[I_{1}\right]=\Phi^{k}\left[\Phi\left[I_{1}\right]\right]=\Phi\left[I_{1}\right]$, it follows 
from the uniqueness that $\Phi\left[I_{1}\right]=I_{1}$ in $E_{0, b}\left(I_{0}\right)$. Moreover, if $\tilde{I} \in E_{0, b}\left(I_{0}\right)$ is such that $\widetilde{I}(t)=0$ for all $t>0$, then

$$
\left.\left.0 \leq \Phi^{k}[\tilde{I}](t) \leq \int_{t-T}^{t} \alpha(\tau) I_{0}\left(\tau-t_{0}\right) P(t, \tau) d \tau \leq r(t)\left\|I_{0}\right\|, \quad \forall t \in\right] 0, b\right],
$$

which implies that, for $k \in \mathbb{N}$ and $t \in] 0, b]$,

$$
\begin{aligned}
I_{1}(t) & =\Phi^{k}\left[I_{1}\right](t) \\
& \leq\left\|\Phi^{k}\left[I_{1}\right]-\Phi^{k}[\tilde{I}]\right\|+r(t)\left\|I_{0}\right\| \\
& \leq \frac{1}{k !}\left\|I_{0}\right\|^{k}\left(\int_{0}^{b} \alpha(\tau) d \tau\right)^{k}\left\|I_{1}\right\|+r(t)\left\|I_{0}\right\| .
\end{aligned}
$$

Taking the limit as $k \rightarrow+\infty$, we obtain

$$
\left.\left.I_{1}(t) \leq r(t)\left\|I_{0}\right\|, \quad \forall t \in\right] 0, b\right] .
$$

Step 2: We consider now $\Phi: E_{b, 2 b}\left(I_{1}\right) \rightarrow E_{b, 2 b}\left(I_{1}\right)$, where $b$ is defined in (3.9). Then, the same arguments of Step 1 hold and we obtain a fixed point $I_{2} \in E_{b, 2 b}\left(I_{1}\right)$ such that

$$
\begin{cases}I_{2}(t)=I_{1}(t), & \forall t \in\left[-T-t_{0}, b\right], \\ I_{2}(t) \leq r(t)\left\|I_{1}\right\|, & \forall t \in] b, 2 b] .\end{cases}
$$

Arguing by induction, we obtain a sequence of functions $\left\{I_{n}\right\}_{n \in \mathbb{N}}$ in $E\left(I_{0}\right)$ which satisfies the following properties: for each $k \in \mathbb{N}$,

$$
\begin{cases}I_{n}(t)=I_{n-1}(t)=\cdots=I_{k}(t), & \left.\forall n \geq k, \quad \forall t \in]-T-t_{0}, k b\right], \\ I_{k}(t) \leq r(t)\left\|I_{k-1}\right\| \leq r(t)\left\|I_{0}\right\|, & \forall t \in](k-1) b, k b] .\end{cases}
$$

If we define

$$
\bar{I}(t)=\lim _{n \rightarrow+\infty} I_{n}(t),
$$

then it is easily seen that $I_{n} \rightarrow \bar{I}$ uniformly on the compacts sets of $\mathbb{R}$. Hence, since

$$
\begin{gathered}
I_{n}(t)=\int_{t-T}^{t} \alpha(\tau) I_{n}\left(\tau-t_{0}\right)\left(1-I_{n}(\tau)\right) P(t, \tau) d \tau, \\
\forall t \in\left[-T-t_{0}, n b\right],
\end{gathered}
$$


we have, after passing to the limit in both sides of (3.15), that $\bar{I}$ is the unique solution of (2.13).

In addition, if $\lim \sup _{t \rightarrow+\infty} r(t)<1$, then there exist $0 \leq \rho<1$ and $M>0$ such that $r(t) \leq \rho, \forall t \geq M$. In particular, it follows from (3.14) that $I_{k_{0}}(t) \leq$ $\rho\left\|I_{k_{0}-1}\right\| \leq \rho\left\|I_{0}\right\|$, for $k_{0}>1+M / b$ and $\left.\left.t \in\right]\left(k_{0}-1\right) b, k_{0} b\right]$.

Hence, we have for $m \in \mathbb{N}$,

$$
\left.\left.\bar{I}(t) \leq \rho^{m+1}\left\|I_{0}\right\|, \quad \forall t \in\right]\left(k_{0}+m-1\right) b,\left(k_{0}+m\right) b\right]
$$

and the conclusion follows with

$$
L=-\frac{\ln \rho}{b} \quad \text { and } \quad C=\rho^{1-k_{0}}
$$

Remark 3.4. As an immediate consequence of the absolute continuity of the Lebesgue integral, it follows that $\bar{I}$ is continuous in the interval $] 0,+\infty[$. In order to assure its continuity in ] $-T-t_{0},+\infty$ [, it suffices to assume that $I_{0}$ is continuous on $\left[-T-t_{0}, 0\right]$ and satisfies the following compatibility condition:

$$
I_{0}(0)=\int_{-T}^{0} \alpha(\tau) I_{0}\left(\tau-t_{0}\right)\left(1-I_{0}(\tau)\right) P(0, \tau) d \tau .
$$

\section{Case 2: "The General Infective Rate"}

In this section we consider the existence and asymptotic behavior of solutions for Eq. (2.13) without the assumption $r(t) \leq 1$. Except for the arguments used to prove the asymptotic behavior, the existence of solutions in this case can be obtained with essentially the same proof as in the previous theorem. Indeed, with the notation introduced before, we have:

Theorem 3.5: Assume $\alpha$ satisfies (3.3). If $I_{0} \in E$, then Eq. (2.13) has a unique solution $\bar{I} \in L_{\text {loc }}^{\infty}\left(-T-t_{0},+\infty\right)$ generated by $I_{0}$.

Proof. We argue as in the proof of Theorem 3.3.

Let $a, b$ be two real numbers such that $-T-t_{0}<a<b$ and consider the set

$$
F_{a, b}\left(I_{0}\right)=\left\{I \in E ; I(\tau)=I_{0}(\tau), \text { for } \tau<a \text {, and } I(\tau)=0 \text {, for } \tau>b\right\} .
$$


It is obvious that $F_{a, b}\left(I_{0}\right)$ is a nonempty closed subset of $E$.

We introduce the operator $\Phi: F_{a, b}\left(I_{0}\right) \rightarrow F_{a, b}\left(I_{0}\right)$ defined by $\Phi[I](t)=I_{0}(t)$ for $t \leq a, \Phi[I]=0$ for $t>b$ and

$$
\left.\left.\Phi[I](t)=\int_{t-T}^{t} \alpha(\tau) I\left(\tau-t_{0}\right)(1-I(\tau)) P(t, \tau) d \tau . \quad \forall t \in\right] a, b\right] .
$$

Repeating the same arguments of steps 1 and 2 in the proof of Theorem 3.3 we show that $\Phi$ has a unique fixed point $I_{n} \in F_{n b,(n+1) b}$, where $b$ is given by (3.9), satisfying the following properties: for each $k \in \mathbb{N}$,

$$
\left.\left.I_{n}(t)=I_{n-1}(t)=\cdots=I_{k}(t), \quad \forall n \geq k, \quad \forall t \in\right]-T-t_{0}, k b\right] .
$$

If we define

$$
\bar{I}(t)=\lim _{n \rightarrow+\infty} I_{n}(t),
$$

then it is easily seen that $I_{n} \rightarrow \bar{I}$ uniformly on the compacts sets of $\mathbb{R}$. Since

$$
I_{n}(t)=\int_{t-T}^{t} \alpha(\tau) I_{n}\left(\tau-t_{0}\right)\left(1-I_{n}(\tau)\right) P(t, \tau) d \tau, \quad \forall t \in\left[-T-t_{0}, n b\right],
$$

we have, after passing to the limit in both sides of (3.17), that $\bar{I}$ is the unique solution of (2.13) generated by $I_{0}$.

Remark 3.6. It is clear that the set $E\left(I_{0}\right)$ is not an invariant set for the operator $\Phi$ if $r(t)$ does not satisfy $r(t) \leq 1$. For instance, let $I(t)=c$ for all $t \geq-T-t_{0}$, where $0 \leq c \leq 1, P(t, \tau)=\phi(t-\tau)$, being $\phi$ the characteristic function of the interval $[0, T]$ and $\alpha(\tau)=1$. Then

$$
\Phi[I](t)=c(1-c) T
$$

and we have $\Phi[I](t)>1$ if $T>\frac{1}{c(1-c)}$. In spite of this, we can show that the solution $\bar{I}$ may belong to $E\left(I_{0}\right)$. This is proved in the next theorem.

We assume that $\phi:[0,+\infty[\rightarrow[0,1]$ is such that
i) $\phi(0)=1$;
ii) $\phi(\xi)=0$ if $\xi \geq T$;
iii) $\phi(\xi)$ is a monotone decreasing function of $\xi$. 
Theorem 3.7. Assume that $\alpha \in E, \alpha(t) \geq 0$ and let $P(t, \tau)=\phi(t-\tau)$, with $\phi$ satisfying (3.18). If $I_{0} \in E$ with $0 \leq I_{0}(t) \leq 1$, then the solution $\bar{I}$ of (2.13) generated by $I_{0}$ satisfies $0 \leq \bar{I}(t) \leq 1, \forall t>-T-t_{0}$.

Proof. We proceed in three steps.

Step 1: In addition to the hypothesis, we assume that

i) $\alpha \in C\left(\left[-T-t_{0},+\infty[), \quad \alpha(t)>0, \quad \forall t \in\left[-T-t_{0},+\infty[\right.\right.\right.$,

ii) $\phi \in C^{1}\left(\left[0,+\infty[), \quad \phi^{\prime}(\xi)<0, \quad \xi \in\right] 0, T[, \quad \phi(0)=1\right.$,

$$
\phi(\xi)=0, \quad \forall \xi \geq T,
$$

We prove firstly that, under (3.19), if $I_{0} \in C\left(\left[-T-t_{0}, 0\right]\right)$ is such that $0<$ $I_{0}(t)<1$ in $\left[-T-t_{0}, 0\right]$ and $\bar{I} \in L_{\mathrm{loc}}^{\infty}$ is the solution of Eq. (2.13) generated by $I_{0}$, then $0<\bar{I}(t)<1$ for all $t>-T-t_{0}$.

Indeed, consider

$$
\Gamma=\{t \in \mathbb{R} ; \bar{I}(t) \geq 1 \text { or } \bar{I}(t) \leq 0\},
$$

which is a closed subset of $\left[0,+\infty\right.$ [. If $\Gamma \neq \emptyset$, let $t_{1}=\inf \Gamma$. Since $\bar{I}(t)=I_{0}(t)$ for $t \leq 0$, then $t_{1}>0$ and $0<\bar{I}(t)<1$ for all $t<t_{1}$.

Suppose that $\bar{I}\left(t_{1}\right)=1$. Since we are assuming (3.19), it follows that $\bar{I}(t)$ is differentiable for $t>0, t \neq t_{0}$. In particular, if $t_{1} \neq t_{0}$, then

$$
\frac{d \bar{I}}{d t}\left(t_{1}\right)=\int_{t_{1}-T}^{t_{1}} \alpha(\tau) \bar{I}\left(\tau-t_{0}\right)(1-\bar{I}(\tau)) \phi^{\prime}\left(t_{1}-\tau\right) d \tau<0
$$

and we can pick up $\delta>0$ such that $\bar{I}\left(t_{1}-\delta\right)>1$, which is a contradiction. Besides,

$$
\begin{aligned}
\lim _{t \rightarrow t_{0}^{-}} \frac{d \bar{I}}{d t}(t)= & \alpha\left(t_{0}\right) I_{0}(0)\left(1-\bar{I}\left(t_{0}\right)\right) \\
& +\int_{t_{0}-T}^{t_{0}} \alpha(\tau) \bar{I}\left(\tau-t_{0}\right)(1-\bar{I}(\tau)) \phi^{\prime}\left(t_{0}-\tau\right) d \tau, \\
\lim _{t \rightarrow t_{0}^{+}} \frac{d \bar{I}}{d t}(t)= & \alpha\left(t_{0}\right) \bar{I}(0)\left(1-\bar{I}\left(t_{0}\right)\right) \\
& +\int_{t_{0}-T}^{t_{0}} \alpha(\tau) \bar{I}\left(\tau-t_{0}\right)(1-\bar{I}(\tau)) \phi^{\prime}\left(t_{0}-\tau\right) d \tau
\end{aligned}
$$


and we have the same contradiction if $t_{1}=t_{0}$.

Suppose that $\bar{I}\left(t_{1}\right)=0$. It follows from the intermediate value theorem for integrals that there exists $\tau \in] t_{1}-T, t_{1}$ [ such that

$$
0=\bar{I}\left(t_{1}\right)=T \alpha(\tau) \bar{I}\left(\tau-t_{0}\right)(1-\bar{I}(\tau)) \phi\left(t_{1}-\tau\right)
$$

and we have also a contradiction.

Therefore, $\Gamma=\emptyset$ and we have the conclusion.

Now let $I_{0} \in E$ such that $0 \leq I_{0}(t) \leq 1$ a.e. in $]-T-t_{0}, 0\left[\right.$ and $\bar{I} \in L_{\text {loc }}^{\infty}$ the solution generated by $I_{0}$.

Since $C\left(\left[-T-t_{0}, 0\right]\right)$ is dense in $L^{1}\left(-T-t_{0}, 0\right)$, it follows that, for a given $\varepsilon>0$, there exists $I_{0}^{\varepsilon}$ continuous such that $0<I_{0}^{\varepsilon}(t)<1$ for $t \in\left[-T-t_{0}, 0\right]$ and

$$
\int_{-T-t_{0}}^{0}\left|I_{0}^{\varepsilon}(t)-I_{0}(t)\right| d t<\varepsilon .
$$

If we denote by $\bar{I}_{\varepsilon}$ the solution of Eq. (2.13) generated by $I_{0}^{\varepsilon}$, then $0<\bar{I}_{\varepsilon}(t)<1$ for $t>0$. Moreover, if $0<t \leq b$, we have

$$
\left|\bar{I}_{\varepsilon}(t)-\bar{I}(t)\right| \leq 3\|\alpha\| \varepsilon+\|\alpha\| \int_{0}^{t}\left|\bar{I}_{\varepsilon}(s)-\bar{I}(s)\right| d s .
$$

From the Gronwall inequality we get

$$
\left|\bar{I}_{\varepsilon}(t)-\bar{I}(t)\right| \leq 3\|\alpha\| \varepsilon \exp (\|\alpha\| b) .
$$

Then, $\bar{I}_{\varepsilon}$ converges to $\bar{I}$ as $\varepsilon \rightarrow 0$, uniformly in $[0, b]$, and we have, in particular, $0 \leq \bar{I}(t) \leq 1 \forall t \in[0, b]$.

Arguing by induction on the intervals $[k b,(k+1) b], k \geq 0$, we conclude that $0 \leq \bar{I}(t) \leq 1$ for $t>0$.

Step 2: In addition to the hypothesis, we assume that

$$
\alpha \in C\left(\left[-T-t_{0},+\infty[), \quad \alpha(t)>0, \quad \forall t \in\left[-T-t_{0},+\infty[.\right.\right.\right.
$$

Let $\bar{I} \in L_{\text {loc }}^{\infty}$ be the solution of Eq. (2.13) generated by $I_{0}$. For $\varepsilon>0$ given, we can construct (for instance, by smoothing step functions) a function $\phi_{\varepsilon}$ satisfying 
(3.19)-(ii) and such that

$$
\int_{0}^{T}\left|\phi_{\varepsilon}(s)-\phi(s)\right| d s<\varepsilon .
$$

Let $\bar{I}_{\varepsilon} \in L_{\text {loc }}^{\infty}$ be the solution of Eq. (2.13) (with $P_{\varepsilon}(t, \tau)=\phi_{\varepsilon}(t-\tau)$ ) generated by $I_{0}$. It follows from step 1 that $0 \leq \bar{I}_{\varepsilon}(t) \leq 1$, for all $t>0$.

Besides, if $0<t \leq b$, we have

$$
\left|\bar{I}_{\varepsilon}(t)-\bar{I}(t)\right| \leq 2\|\alpha\| \varepsilon+\|\alpha\| \int_{0}^{t}\left|\bar{I}_{\varepsilon}(s)-\bar{I}(s)\right| d s .
$$

Hence, the Gronwall inequality implies $\bar{I}_{\varepsilon} \rightarrow \bar{I}$ as $\varepsilon \rightarrow 0$, uniformly on ] 0,b], and we have the same conclusion as in step 1.

Step 3: Let $\bar{I}$ be the solution of Eq. (2.13) generated by $I_{0}$. Since we are assuming $\alpha \in E$, for each $\varepsilon>0$, we can construct a function $\alpha_{\varepsilon}$ satisfying (3.21) and such that

$$
\int_{(n-1) b}^{n b}\left|\alpha_{\varepsilon}(s)-\alpha(s)\right| d s<\varepsilon, \quad \forall n \in \mathbb{N} .
$$

Then, the same arguments of step 2 allows to show that $0 \leq \bar{I} \leq 1$ for all $t>0$ and this completes the proof.

Remark 3.8. Concerning the asymptotic behavior of the solutions of Eq. (2.13), it should be noticed that, if $r(t)$ is defined by (3.4) and satisfies

$$
\lim _{t \rightarrow+\infty} r(t)=\rho_{T}
$$

we expect to have the following global asymptotics for the solution $\bar{I}$ generated by $0 \leq I_{0} \leq 1$ :

$$
\lim _{t \rightarrow+\infty} \bar{I}(t)= \begin{cases}0 & \text { if } \rho_{T}<1, \\ 1-1 / \rho_{T} & \text { if } \rho_{T} \geq 1 .\end{cases}
$$

Actually, $\rho_{T}<1$ is a particular case of Slow Infective Rate and the asymptotic behavior is obtained in Theorem 3.3. 
Although it seems to be difficult to prove the global behavior (3.23) in general, some simple evidences can be obtained in the special case where $P(t, \tau)=$ $\phi(t-\tau)$, with $\phi$ satisfying (3.18) and $\alpha(t) \equiv \alpha$ is constant. Indeed, it is easily seen that, in this case we have $\rho_{T}=\alpha \int_{0}^{T} \phi(\tau) d \tau$ and

$$
I(t) \equiv 0 \quad \text { and } \quad I(t) \equiv 1-1 / \rho_{T}
$$

are the only stationary solutions of Eq. (2.13).

Assuming that $I_{\infty}$ is one of the stationary solutions of Eq. (2.13) and considering $\bar{I}(t)=I_{\infty}+X(t)$, the solution generated by $I_{0}(t)=I_{\infty}+X_{0}(t)$, we have for $t>0$

$$
\begin{aligned}
X(t)= & \alpha\left(1-I_{\infty}\right) \int_{t-T}^{t} X\left(\tau-t_{0}\right) \phi(t-\tau) d \tau \\
& -\alpha I_{\infty} \int_{t-T}^{t} X(\tau) \phi(t-\tau) d \tau \\
& -\alpha \int_{t-T}^{t} X\left(\tau-t_{0}\right) X(\tau) \phi(t-\tau) d \tau .
\end{aligned}
$$

Discarding all but linear terms in Eq. (3.25), we obtain the linear integral equation

$$
X(t)=\mathcal{L}[X](t)
$$

where

$$
\begin{aligned}
\mathcal{L}[X](t)= & \alpha\left(1-I_{\infty}\right) \int_{t-T}^{t} X\left(\tau-t_{0}\right) \phi(t-\tau) d \tau \\
& -\alpha I_{\infty} \int_{t-T}^{t} X(\tau) \phi(t-\tau) d \tau .
\end{aligned}
$$

The behavior of solutions of (3.26) can be studied in terms of its associated characteristic roots $\lambda \in \mathbb{C}$. More precisely, assuming $X(t)=e^{\lambda t}$ and substituting in (3.26) we obtain the characteristic equation

$$
\alpha\left(1-I_{\infty}\right) e^{-\lambda t_{0}} \int_{0}^{T} e^{-\lambda \tau} \phi(\tau) d \tau-\alpha I_{\infty} \int_{0}^{T} e^{-\lambda \tau} \phi(\tau) d \tau=1
$$

Lemma 3.9. We assume that $r(t)$ satisfies (3.22) with $\rho_{T}>1$. Then the characteristic equation (3.27) has a unique real solution $\bar{\lambda}$ satisfying:

a) $\bar{\lambda}>0$ if $I_{\infty}=0$;

b) $\bar{\lambda}<0$ if $I_{\infty}=1-1 / \rho_{T}$. 
Proof. Let $f: \mathbb{R} \rightarrow \mathbb{R}$ be the function defined by

$$
f(\lambda)=\alpha\left[\left(1-I_{\infty}\right) e^{-\lambda t_{0}}-I_{\infty}\right] \int_{0}^{T} e^{-\lambda \tau} \phi(\tau) d \tau .
$$

If $I_{\infty}=0$, then (3.28) is given by

$$
f(\lambda)=\alpha e^{-\lambda t_{0}} \int_{0}^{T} e^{-\lambda \tau} \phi(\tau) d \tau .
$$

and it is easily seen that $f(\lambda)$ is positive, monotone decreasing and satisfies:

$$
\lim _{\lambda \rightarrow-\infty} f(\lambda)=+\infty, \quad \lim _{\lambda \rightarrow+\infty} f(\lambda)=0 .
$$

Since $f(\lambda)$ is a continuous function, there exists a unique $\bar{\lambda}$ satisfying equation (3.27). Since $f(0)=\rho_{T}>1$, we conclude that $\bar{\lambda}>0$ and a) is proved.

If $I_{\infty}=1-1 / \rho_{T}$, then (3.28) is given by

$$
f(\lambda)=\alpha\left[\frac{1}{\rho_{T}}\left(e^{-\lambda t_{0}}+1\right)-1\right] \int_{0}^{T} e^{-\lambda \tau} \phi(\tau) d \tau
$$

and the limits in (3.29) hold. Moreover, $f(\lambda)$ is positive and monotone decreasing in the interval

$$
-\infty<\lambda \leq \ln \left(\frac{1}{\rho_{T}-1}\right)^{1 / t_{0}}
$$

and negative elsewhere. Since it is continuous, there exists a unique

$$
\bar{\lambda}<\ln \left(\frac{1}{\rho_{T}-1}\right)^{1 / t_{0}}
$$

such that $f(\bar{\lambda})=1$. In order to prove that $\bar{\lambda}<0$, we distinguish two cases.

If $\rho_{T} \geq 2$, then $\ln \left(1 /\left(\rho_{T}-1\right)\right)^{1 / t_{0}} \leq 0$ and the conclusion follows from (3.30).

If $1<\rho_{T}<2$, then $0<f(0)=2-\rho_{T}<1=f(\bar{\lambda})$ and the conclusion follows from the monotonicity of $f$. 
Remark 3.10. The condition a) in Lemma 3.9 is sufficient to assure the instability of the stationary solution $I_{\infty}=0$ in the case $\rho_{T}>1$. On the other hand, although the condition $b$ ) is necessary to have the local asymptotic stability of $I_{\infty}=1-1 / \rho_{T}$, we do not have a precise characterization of the possible complex roots of Eq. (3.27) (see [3, 8, 7]).

The next theorem concerns the continuity of solutions of Eq. (2.13) with respect to the parameter $t_{0}$.

Theorem 3.11. Assume $\alpha$ and $P$ satisfying (3.3) and (2.2) respectively. Let $\left\{t_{n}\right\}_{n \in \mathbb{N}}$ be a sequence of positive real numbers such that $t_{n} \rightarrow t_{0}\left(t_{0}>0\right)$ as $n \rightarrow+\infty$. Let $I_{0} \in L^{\infty}(-T-\delta, 0)$ be a function satisfying $0 \leq I_{0}(t) \leq 1$, where $\delta=\sup \left\{t_{n} ; n \in \mathbb{N}\right\}$. If $\bar{I}_{n} \in E\left(I_{0}\right)$ is the solution of

$$
I_{n}(t)=\int_{t-T}^{t} \alpha(\tau) I_{n}\left(\tau-t_{n}\right)\left(1-I_{n}(\tau)\right) P(t, \tau) d \tau
$$

generated by $I_{0}$, then $\bar{I}_{n} \rightarrow \bar{I}$ uniformly on the compacts of $[0,+\infty[$, where $\bar{I}$ is the solution of (2.13) generated by $I_{0}$.

Proof. We have from the previous results that, for each $n \in \mathbb{N}$, there exists a unique solution $\bar{I}_{n}$ of Eq. (3.31) generated by $I_{0}$, such that $0 \leq \bar{I}_{n}(t) \leq 1$ a.e. in the interval ] $-T-\delta,+\infty$ [. In particular, it follows that $\left\{\bar{I}_{n}\right\}_{n \in \mathbb{N}}$ is a bounded subset of the Banach space $C([0, R] ; \mathbb{R})$, for each $R>0$.

Moreover, for $t>0$ and $h>0$ we have

$$
\left|\bar{I}_{n}(t+h)-\bar{I}_{n}(t)\right| \leq \int_{t}^{t+h}(\alpha(\tau)+\alpha(\tau+T)) d \tau
$$

and the absolute continuity of the Lebesgue integral implies that $\left\{\bar{I}_{n}\right\}_{n \in \mathbb{N}}$ is an equicontinuous subset of $C([0, R] ; \mathbb{R})$.

Fixing $R>0$, we have from the Arzelà-Ascoli theorem that there exist a subsequence $\left\{n_{k}\right\}_{k \in \mathbb{N}}$ and a function $I_{R} \in C([0, R] ; \mathbb{R})$ such that

$$
\lim _{k \rightarrow \infty} \bar{I}_{n_{k}}=I_{R} \quad \text { uniformly on } \quad[0, R] .
$$


Defining $I_{R}(\tau)=I_{0}(\tau)$ for $\tau<0$, we can write for $t>0$

$$
\begin{aligned}
\bar{I}_{n_{k}}(t)= & \int_{t-T}^{t} \alpha(\tau)\left[\bar{I}_{n_{k}}\left(\tau-t_{n_{k}}\right)-I_{R}\left(\tau-t_{n_{k}}\right)\right]\left(1-\bar{I}_{n_{k}}(\tau)\right) P(t, \tau) d \tau \\
& +\int_{t-T}^{t} \alpha(\tau) I_{R}\left(\tau-t_{n_{k}}\right)\left(1-\bar{I}_{n_{k}}(\tau)\right) P(t, \tau) d \tau .
\end{aligned}
$$

Passing to the limit as $k \rightarrow \infty$ in both sides of (3.32) we have

$$
I_{R}(t)=\int_{t-T}^{t} \alpha(\tau) I_{R}\left(\tau-t_{0}\right)\left(1-I_{R}(\tau)\right) P(t, \tau) d \tau
$$

and we conclude from the uniqueness of solutions (see Lemma 3.1) that the full sequence $\left\{\bar{I}_{n}\right\}$ converges uniformly to $I_{R}$. Since $R>0$ is fixed arbitrarily, the proof is finished.

Remark 3.12. Although we are tacitly assuming that $t_{0}>0$ in Theorem 3.11, it is interesting to point out that the conclusion is also true in the case $t_{n} \rightarrow 0^{+}$. Indeed, the Eq. (2.13) with $t_{0}=0$ has a unique global solution generated by $0 \leq I_{0} \leq 1$ and the same arguments in the proof Theorem 3.11 hold.

\section{Numerical results and conclusions}

In order to perform numerical calculations in the absence of previous statistical data, we have to propose a certain infection-rate function $g(t)$ for the interval $-T-t_{0} \leq t \leq 0$. It has not to be a realistic function, since our purpose in this section is to illustrate the salient features of the time evolution process and not to reproduce or to predict actual situations in a realistic fashion. For this purpose, we consider the simplest case of a constant infection-rate function $g(t)=I_{0} / T$ for $t \in\left[-T-t_{0}, 0\right]$, where $I_{0}$ represents the density of infective computers at $t=0$. Furthermore, the contact-rate factor $\alpha(\tau)$ was also considered as a constant and the probability of a computer infected at $\tau$ to remain infectious at $t$ was chosen as $P(t, \tau)=\phi(t-\tau)$, where $\phi$ is the characteristic function of the interval [0, $T$ [ , or equivalently, $P(t, \tau)=H(t-\tau)-H(t-\tau-T)$, where $H(\xi)$ is the Heaviside step function.

Figure 1 shows a typical "slow infective rate case" for which, under our oversimplified considerations, $\alpha T<1$. The graph presents singularities in the 
interval $\left[0, t_{0}+T\right]$ due to the arbitrary choice of initial data but, as it can be seen, the infection process is self controlled, tending to disappear for increasing $t$ values. On the other hand, Fig. 2, 3 and 4 show the more complex situation of typical "fast infective rate cases"' $\alpha T>1$. In each case, strong (but damped) oscillations would appear for $\alpha T$ large enough. Nevertheless, an equilibrium point $I_{\infty}=(\alpha T-1) / \alpha T$ is attained for $t \rightarrow+\infty$, characterizing an endemic situation.

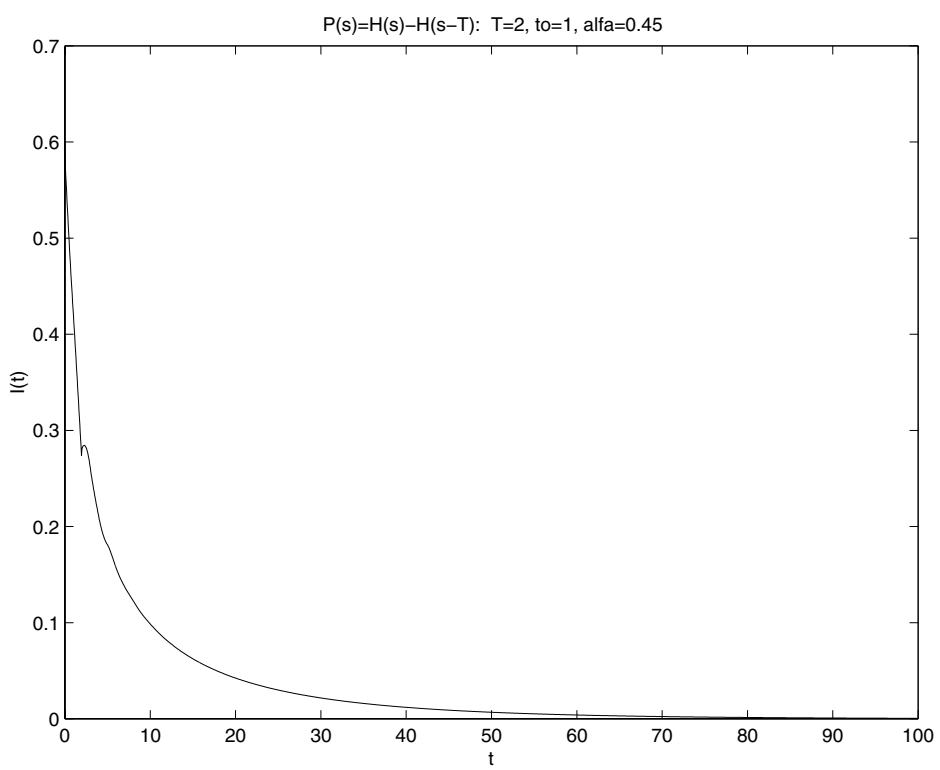

Figure 1 - Density of infected computers as a function of time in a slow infective rate case. We consider $g(t)=I_{0} / T=0.3$ for $t \in\left[-T-t_{0}, 0\right], \alpha=0.45, t_{0}=1, T=2$ and $P(t, \tau)=\phi(t-\tau)$, where $\phi(\xi)$ is the characteristic function of [0,T[. Notice that in this case, the infection process affecting the system tends to disappear for $t$ large enough, in agreement with the previous results.

As it was shown in the previous section, the stationary solution $I_{\infty}=0$ is an asymptotically stable solution for $\rho_{T}=\alpha T<1$. This means that when such a condition holds, the introduction of a few infected computers into an infectivefree population won't give rise to an epidemic outbreak and also no endemic situations will be developed, i.e., the infection will vanish along the time.

We consider that, in spite of its inherent limitations because of the strong 
hypothesis included, the present work constitutes a first attempt to explain the virtual virus propagation process in a system of interacting computers and reveals some features. These result, we hope, could stimulate future researches in such a current problem. At present, we are engaged in the developing of more sophisticated models in order to take into account immunity factors (the existence of vaccines) as well as to include the spatial dependence of the infectious process. Results in this direction will be published elsewhere.

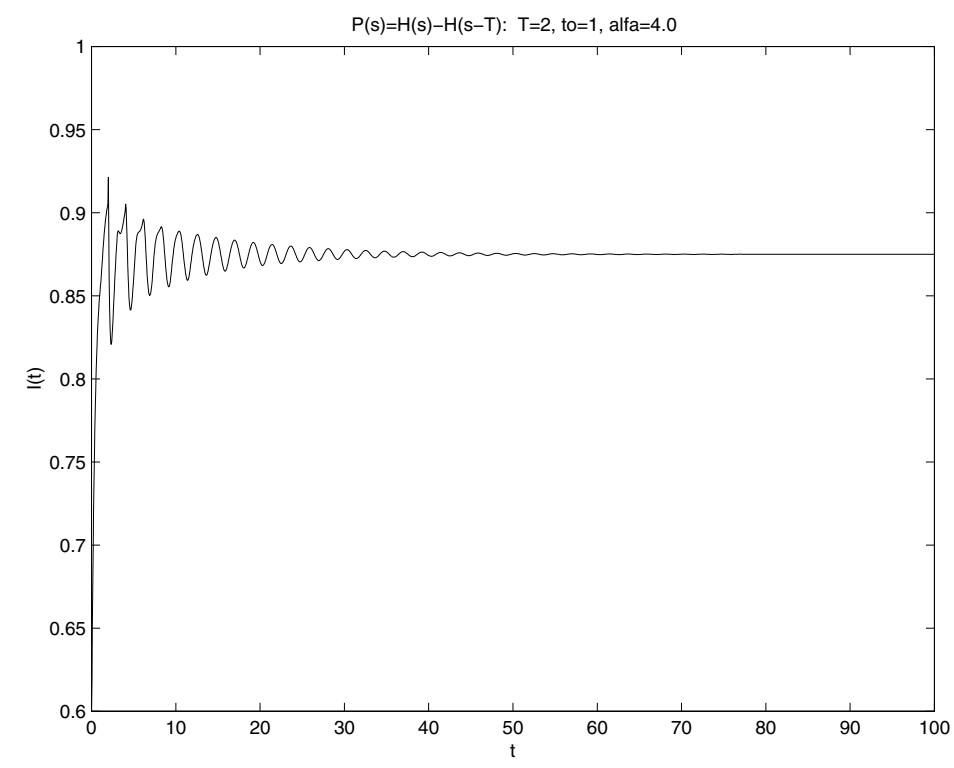

Figure 2 - Density of infected computers as a function of time in a fast infective rate case. Here $g(t)=I_{0} / T=0.3$ for $t \in\left[-T-t_{0}, 0\right], \alpha=4.0, P(t, \tau), t_{0}$ and $T$ are the same as in Figure 1. An equilibrium endemic situation $\left(I_{\infty}=(\alpha T-1) / \alpha T\right)$ is attained when $t \rightarrow \infty$, as can be seen from the figure. This last behavior seems to be a general one for $\alpha T>1$, because using different parameters in numerical calculations we have always obtained such a result.

\section{Acknowledgments}

The authors are grateful to Dr. Felipe Acker (Instituto de Matemática, UFRJ) for suggesting the subject of this paper and the referees for corrections. 

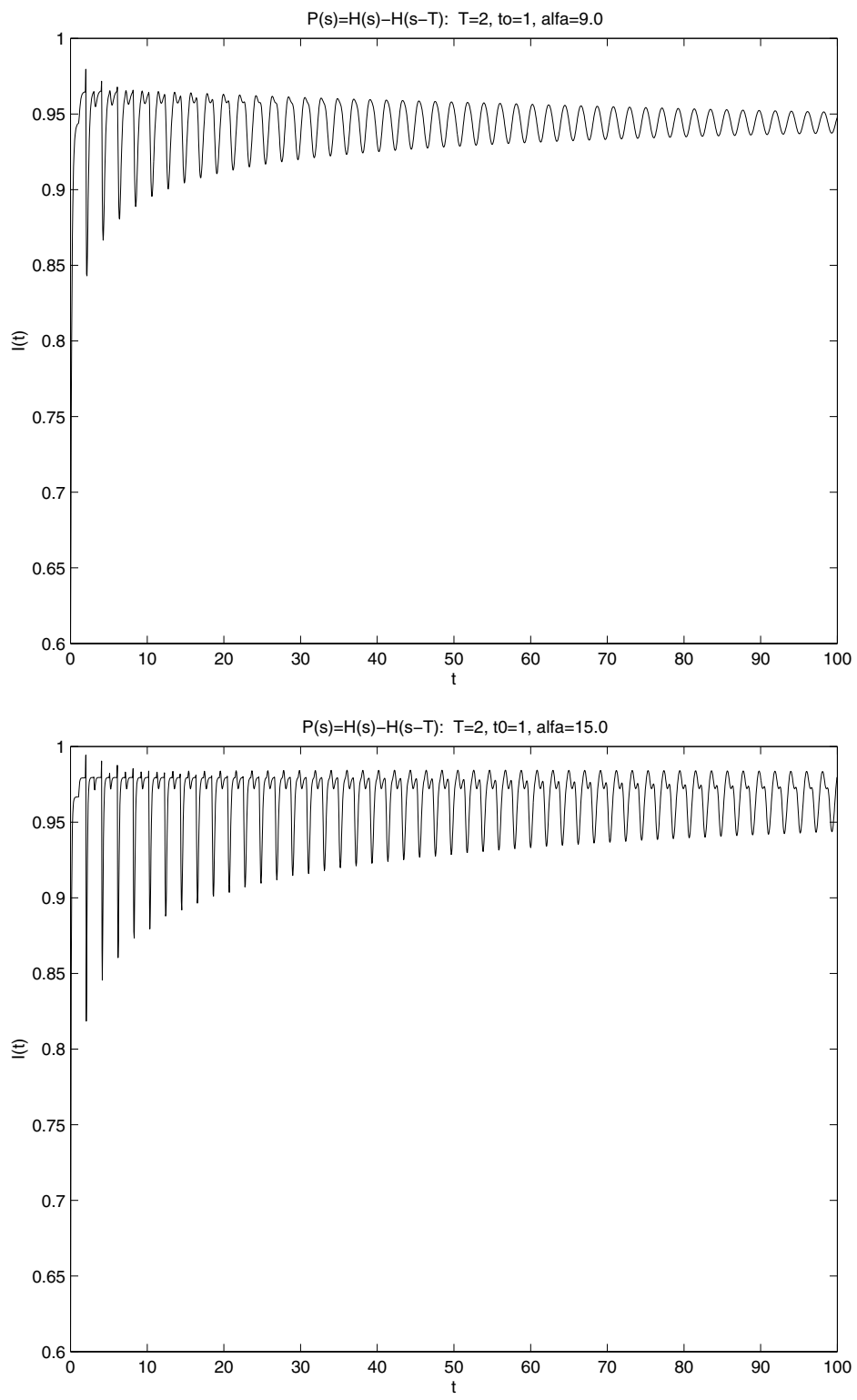

Figure 3 - The same as in Figure 2, with $\alpha=9.0$ and $\alpha=15.0$ respectively. 


\section{REFERENCES}

[1] N.J.T. Bailey, The Mathematical Theory of Infectious Diseases and its Applications, Griffin, London (1975).

[2] C.T.H. Bakes and G.F. Miller, Treatment of Integral Equations by Numerical Methods, Academic Press (1982).

[3] R. Bellmann and K.L. Cooke, Differential-Difference Equations, Math. in Sciences and Engineering, Academic Press (1963).

[4] V. Capasso, Mathematical Structure of Epidemic systems, Springer-Verlag (1993).

[5] J.A. Cochran, The Analysis of Linear Integral Equations, MacGraw-Hill, Meth. Appl. Math., (1972).

[6] K.L. Cooke and J.A. Yorke, Some equations modelling growth processes and gonorrhea epidemics, Mathematical Biosciences, 16 (1973), pp. 75-101.

[7] R.D. Driver, Ordinary and Delay Differential Equations, Applied Mathematical Sciences, 3 (1977), Springer-Verlag.

[8] J. Hale, Theory of Functional Differential Equations, Applied Mathematical Sciences, 3 (1977), Springer-Verlag. 